\title{
A new chaotic behavior of a general model of the Henon map
}

\author{
Ahmed MA El-Sayed ${ }^{1 *}$, Zaki FE El-Raheem² and Sanaa M Salman²
}

${ }^{\text {*Correspondence: }}$ amasayed@gmail.com

'Department of Mathematics, Faculty of Science, Alexandria University, Alexandria, Egypt Full list of author information is available at the end of the article

\begin{abstract}
In this paper we are concerned with a general form of the Henon map as a retarded functional equation. The existence of a unique solution is proved. The continuous dependence of the solution and the local stability of fixed points are investigated. Chaos, bifurcation and chaotic attractor of the resulting system are discussed. In addition, we compare our results with the discrete dynamical system of the Henon map.
\end{abstract}

Keywords: retarded functional equation; Henon map; fixed points; existence; uniqueness; bifurcation; chaos; chaotic attractor

\section{Introduction}

Discontinuous (sectionally continuous) dynamical systems have been defined as a problem of retarded functional equation and studied in [1-10]. The generalized time-delayed Henon map was introduced in $[11,12]$. In this work we study the discontinuous (sectionally continuous) dynamical system of the Henon map as a problem of retarded functional equation with two different delays

$$
x(t)=1+\beta x\left(t-r_{1}\right)-\alpha x^{2}\left(t-r_{2}\right), \quad r_{1}, r_{2}>0, t \in(0, T],
$$

with

$$
x(t)=x_{0}, \quad t \leq 0,
$$

where $\alpha>0$ and $|\beta|<1$.

The existence of a unique continuous dependence solution is proved. The local stability of fixed points is studied. The chaos, bifurcation and chaotic attractor are discussed. Comparison with the corresponding discrete dynamical system of the Henon map

$$
x_{n+1}=1+\beta x_{n-1}-\alpha x_{n}^{2}, \quad n=1,2,3,4, \ldots,
$$

is given.

Let $f:[0, T] \times \mathbb{R}^{n} \rightarrow \mathbb{R}$ and $r_{1}, r_{2}, \ldots, r_{k} \in \mathbb{R}^{+}$.

Consider the problem of retarded functional equation

$$
x(t)=f\left(x\left(t-r_{1}\right), x\left(t-r_{2}\right), \ldots, x\left(t-r_{k}\right)\right), \quad t \in(0, T],
$$

O2014 El-Sayed et al.; licensee Springer. This is an Open Access article distributed under the terms of the Creative Commons Attribution License (http://creativecommons.org/licenses/by/2.0), which permits unrestricted use, distribution, and reproduction in any medium, provided the original work is properly cited. 
with the initial condition

$$
x(\tau)=\phi(\tau), \quad \tau \leq 0 .
$$

If $T$ is a positive integer, $r_{k}=k, \phi(0)=x_{0}$, and $t=n=1,2,3, \ldots$, then problem (1.4)-(1.5) will be the discrete dynamical system

$$
\begin{aligned}
& x_{n}=f\left(x_{n-1}, x_{n-2}, \ldots, x_{n-k}\right), \quad n=1,2,3, \ldots, T, \\
& x(0)=x_{0} .
\end{aligned}
$$

This shows that discrete dynamical system (1.6)-(1.7) is a special case of the problem of retarded functional equation (1.4)-(1.5).

Consider also the singularly perturbed differential difference equation [13]

$$
\epsilon x^{\prime}(t)=-x(t)+f(x(t-1)),
$$

and the singularly perturbed delay differential equation [14]

$$
\epsilon x^{\prime}(t)=a_{0} x(t)+\sum_{j=1}^{k} x\left(t-m_{j}\right),
$$

$m_{j} \geq 0, m_{j} \in \mathbb{Z}, j=1, \ldots, k$.

The limiting cases as $\epsilon \rightarrow 0$ of (1.8) and (1.7) are special cases of retarded functional equation (1.4)-(1.5).

Let $t \in(0, r]$, then $t-r \in(-r, 0]$, the solution of (1.4)-(1.5) (by the method of steps as in $[13-16])$ is given by

$$
x(t)=x_{1}(t)=f(\phi(0)), \quad t \in(0, r] .
$$

For $t \in(r, 2 r]$, then $t-r \in(0, r]$, the solution of (1.4)-(1.5) is given by

$$
x(t)=x_{2}(t)=f\left(x_{1}(t)\right)=f(f(\phi(0)))=f^{2}(\phi(0)), \quad t \in(r, 2 r] .
$$

Repeating the process we can easily deduce the solution of (1.4)-(1.5) which is given by

$$
x(t)=x_{n}(t)=f^{n}(\phi(0)), \quad t \in((n-1) r, n r],
$$

which is continuous on each subinterval $((k-1) r, k r), k=1,2,3, \ldots, n$, but

$$
\lim _{t \rightarrow k r^{+}} x_{(k+1) r}(t)=f^{k+1}(\phi(0)) \neq x_{k r},
$$

which implies that the solution of problem (1.4)-(1.5) is discontinuous (sectionally continuous) on $(0, T]$.

Now we have the following definitions.

Definition 1 The discontinuous (sectionally continuous) dynamical system is the problem of retarded functional equation (1.4)-(1.5). 
Definition 2 The fixed points of discontinuous (sectionally continuous) dynamical system (1.4)-(1.5) are the solution of the equation

$$
x(t)=f(t, x, x, \ldots, x) .
$$

Remark 1 We should notice that the difference equations representing the Henon map in its different cases,

$$
\begin{aligned}
& x_{n+1}=1+\beta x_{n-1}-\alpha x_{n-1}^{2}, \quad n=1,2,3,4, \ldots, \\
& x_{n+1}=1+\beta x_{n}-\alpha x_{n-1}^{2}, \quad n=1,2,3,4, \ldots, \\
& x_{n+1}=1+\beta x_{n}-\alpha x_{n}^{2}, \quad n=1,2,3,4, \ldots,
\end{aligned}
$$

are just special cases of our problem (1.1)-(1.2).

\section{Existence and uniqueness}

Now consider the discontinuous (sectionally continuous) dynamical system of the Henon map (1.1)-(1.2). The existence of a unique solution as well as the continuous dependence of the solution on the initial data are proved. We study also the continuous dependence of the solution on the parameter $\alpha$.

Let $L^{1}=L^{1}[0, T], T<\infty$, be the class of Lebesgue integrable functions on $[0, T]$ with the norm

$$
\|f\|=\int_{0}^{T}|f(t)| d t, \quad f \in L^{1} .
$$

Let $D=\left\{x \in \mathbb{R}:|x|<k, k=\frac{1}{1-|\beta|}\right\} \subset L^{1}[0, T]$.

Definition 3 By a solution of problem (1.1)-(1.2) we mean that the function $x \in L^{1}$ satisfies problem (1.1)-(1.2).

Theorem 1 The sufficient condition for the existence of a unique solution of problem (1.1)-

(1.2) is $|\beta|+2 \alpha k<1$.

Proof Define the operator $F: L^{1} \rightarrow L^{1}$ on $D$ by

$$
F x(t)=1+\beta x\left(t-r_{1}\right)-\alpha x^{2}\left(t-r_{2}\right),
$$

then

$$
|F x(t)| \leq 1+\beta|x|<1+|\beta| k \leq k .
$$

This proves that $F: D \rightarrow D$.

Now, for $x, y \in D$, we have

$$
|F x-F y| \leq|\beta|\left|x\left(t-r_{1}\right)-y\left(t-r_{1}\right)\right|+\alpha\left|x^{2}\left(t-r_{2}\right)-y^{2}\left(t-r_{2}\right)\right| .
$$


Thus we can get

$$
\|F x-F y\| \leq|\beta|\|x-y\|+2 \alpha k\|x-y\| .
$$

If $M=|\beta|+2 \alpha k<1$, then

$$
\|F x-F y\| \leq M\|x-y\| .
$$

So, problem (1.1)-(1.2) has, on $D$, a unique solution $x \in L^{1}$.

\section{Continuous dependence on the initial conditions}

Theorem 2 The solution of discontinuous (sectionally continuous) dynamical system (1.1)(1.2) is continuously dependent on the initial data.

Proof Let $x(t)$ and $x^{*}(t)$ be the solutions of dynamical system (1.1)-(1.2) and the dynamical system of equation (1.1) with the initial data

$$
x(0)=x_{0}^{*}
$$

Then

$$
\left|x(t)-x^{*}(t)\right| \leq|\beta|\left|x\left(t-r_{1}\right)-x^{*}\left(t-r_{1}\right)\right|+\alpha\left|x^{2}\left(t-r_{2}\right)-x^{* 2}\left(t-r_{2}\right)\right|,
$$

and we can get

$$
\left\|x(t)-x^{*}(t)\right\| \leq\left(r_{1}|\beta|+r_{2} \alpha\right)\left|x_{0}-x_{0}^{*}\right|+(|\beta|+2 \alpha k)\left\|x-x^{*}\right\| .
$$

This implies that

$$
\left\|x(t)-x^{*}(t)\right\| \leq \frac{r_{1} \beta+r_{2} \alpha}{1-|\beta|-2 \alpha k}\left|x_{0}-x_{0}^{*}\right| .
$$

That is,

$$
\left|x-x^{*}\right| \leq \delta \quad \Rightarrow \quad\left\|x(t)-x^{*}(t)\right\| \leq \epsilon=\frac{r_{1} \beta+r_{2} \alpha}{1-|\beta|-2 \alpha k} \delta .
$$

\section{Continuous dependence on the parameter $\alpha$}

Theorem 3 The solution of discontinuous (sectionally continuous) dynamical system (1.1)(1.2) is continuously dependent on the parameter $\alpha$.

Proof Let $x(t)$ and $x^{*}(t)$ be the solutions of dynamical system (1.1)-(1.2) and the dynamical system

$$
x(t)=1+\beta x\left(t-r_{1}\right)-\alpha^{*} x^{2}\left(t-r_{2}\right),
$$

with the initial data (1.2), then

$$
\left|x(t)-x^{*}(t)\right| \leq\left|\alpha x^{* 2}\left(t-r_{2}\right)-\alpha^{*} x^{* 2}\left(t-r_{2}\right)\right|,
$$


which gives

$$
\left\|x(t)-x^{*}(t)\right\| \leq|\beta|\left\|x-x^{*}\right\|+2 \alpha\left\|x-x^{*}\right\|+k\left|\alpha-\alpha^{*}\right|\left\|x^{*}\right\| .
$$

This implies that

$$
\left\|x(t)-x^{*}(t)\right\| \leq \frac{\left\|x^{*}\right\|}{1-|\beta|-2 \alpha k}\left|\alpha-\alpha^{*}\right| .
$$

That is,

$$
\left|\alpha-\alpha^{*}\right| \leq \delta \Rightarrow\left\|x(t)-x^{*}(t)\right\| \leq \epsilon=\frac{\left\|x^{*}\right\|}{1-\beta-2 \alpha k} \delta .
$$

\section{Fixed points and stability}

Exactly like its discrete counter part, dynamical system (1.1)-(1.2) has two fixed points which are the solutions of the equation

$$
x=1+\beta x-\alpha x^{2} .
$$

So, we have

$$
\begin{aligned}
& \left(x_{\mathrm{fix}}\right)_{1}=\frac{(\beta-1)+\sqrt{(1-\beta)^{2}+4 \alpha}}{2 \alpha}, \\
& \left(x_{\mathrm{fix}}\right)_{2}=\frac{(\beta-1)-\sqrt{(1-\beta)^{2}+4 \alpha}}{2 \alpha} .
\end{aligned}
$$

Obviously, they exist only for $(1-\beta)^{2}+4 \alpha \geq 0$ [15]. To determine the stability of a fixed point, consider a small perturbation from the fixed point by letting

$$
x(t)=x_{\mathrm{fix}}+\epsilon_{0} \lambda^{t} .
$$

Substituting in (2.2) we get

$$
x_{\mathrm{fix}}+\epsilon_{0} \lambda^{t}=1+\beta\left(x_{\mathrm{fix}}+\epsilon_{0} \lambda^{t-r_{1}}\right)-\alpha\left(x_{\mathrm{fix}}+\epsilon_{0} \lambda^{t-r_{2}}\right)^{2},
$$

which implies that the fixed points are asymptotically stable if all roots of the equation

$$
1=\beta \lambda^{-r_{1}}-2 \alpha x_{\mathrm{fix}} \lambda^{-r_{2}}
$$

satisfy $|\lambda|<1$, where $x(t)=x_{\mathrm{fix}}+\epsilon \lambda^{t}$. Here we study three cases:

- $r_{1}=r_{2}$ then $\left(x_{\text {fix }}\right)_{1}$ is stable if $(1-\beta)^{2}+4 \alpha<4$.

- $r_{2}=2 r_{1}$ then $\left(x_{\mathrm{fix}}\right)_{1}$ is stable if $2 \beta+4 \alpha<3$.

- $r_{1}=2 r_{2}$ then $\left(x_{\mathrm{fix}}\right)_{1}$ is stable if $\alpha<\frac{3}{4}(1-\beta)^{2}$.

In all the simulations, $r_{1}$ and $r_{2}$ are rationally dependent.

Figure 1 illustrates the trajectories of (1.3), while Figure 2 illustrates the trajectories of (1.1). 


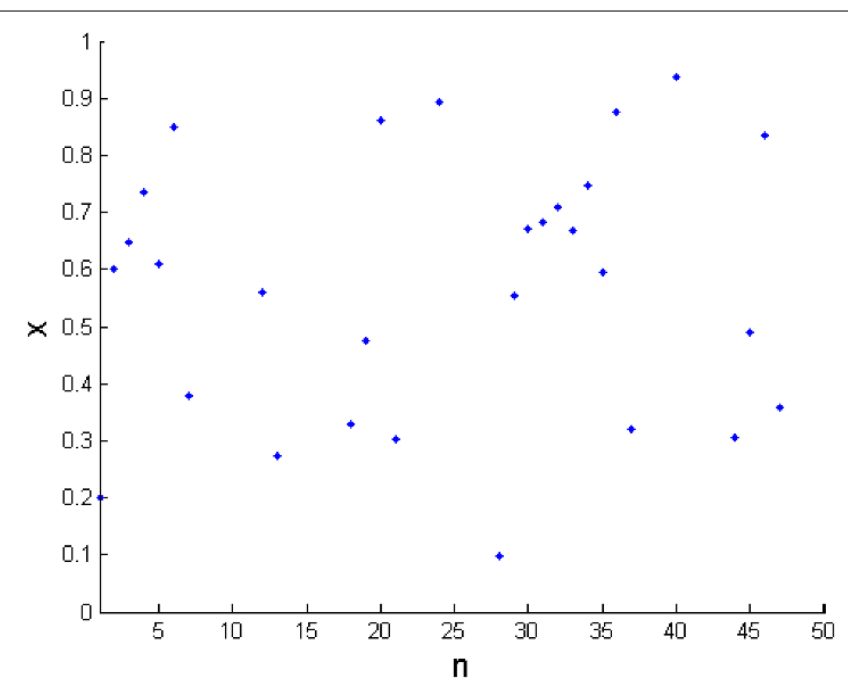

Figure 1 Trajectories of (1.3) with $\alpha=1.2$ and $\beta=0.4$.

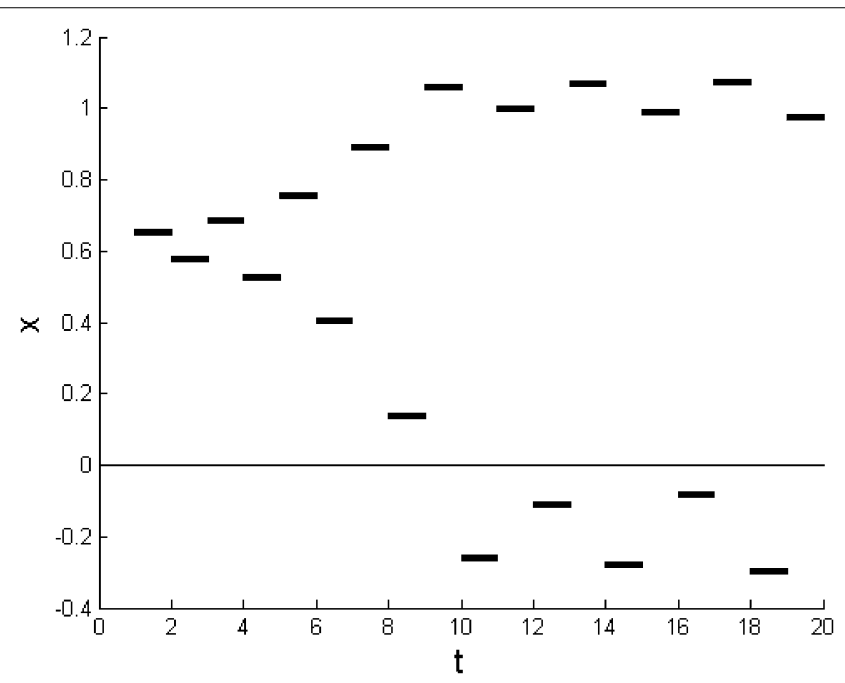

Figure 2 Trajectories of (1.1) with $\alpha=1.2, \beta=0.4$, and $r_{1}=r_{2}=1$.

\section{Bifurcation and chaos}

In this section we show, by numerical experiments illustrated by bifurcation diagrams, that the dynamical behavior of discontinuous (sectionally continuous) dynamical system (1.1)-(1.2) is completely affected by the change in both $r$ and $T$ [17]. We consider three cases for different delays $r_{1}$ and $r_{2}$ as follows.

- Case 1: $r_{1}>r_{2}$.

Let $\beta=0.3$ be fixed and vary $\alpha$ from 0 to 1.4 with step size 0.001 and the initial condition $\left(x_{0}, y_{0}\right)=(0.3,0)$.

Take $r_{1}=2$ and $r_{2}=1$ and $t \in[0,150]$ in (1.1)-(1.2) (Figure 3).

Take $r_{1}=0.50$ and $r_{2}=0.25$ and $t \in[0,38]$ in (1.1)-(1.2) (Figure 4).

Take $r_{1}=0.3$ and $r_{2}=0.1$ and $t \in[0,20]$ in (1.1)-(1.2) (Figure 5).

Take $r_{1}=0.25$ and $r_{2}=0.15$ and $t \in[0,15]$ in (1.1)-(1.2) (Figure 6). 


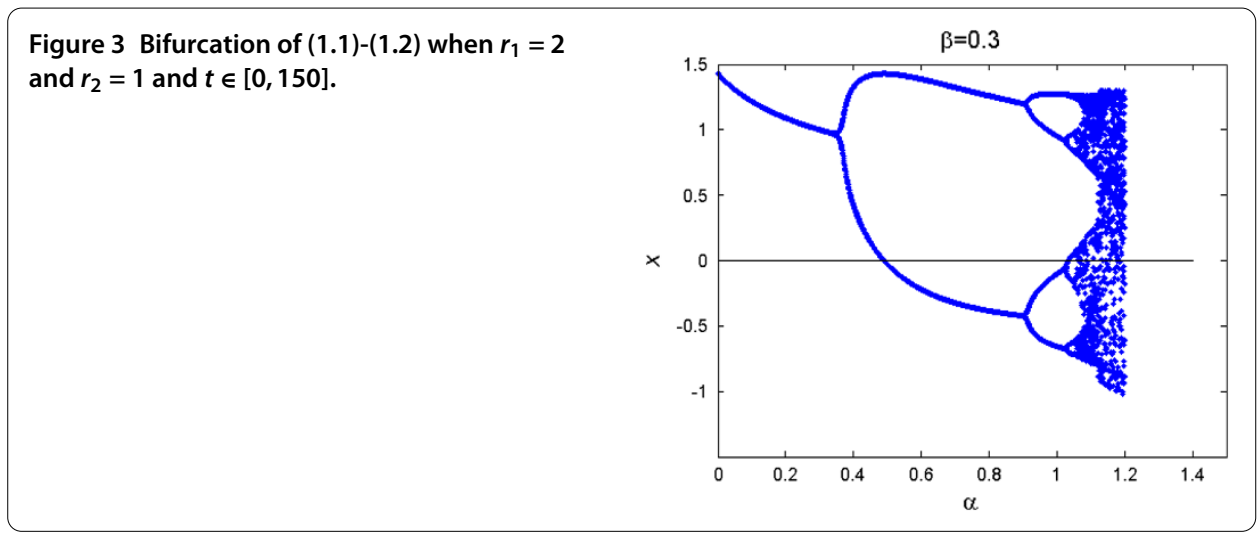

Figure 4 Bifurcation of (1.1)-(1.2) when $r_{1}=0.50$ and $r_{2}=0.25$ and $t \in[0,38]$.

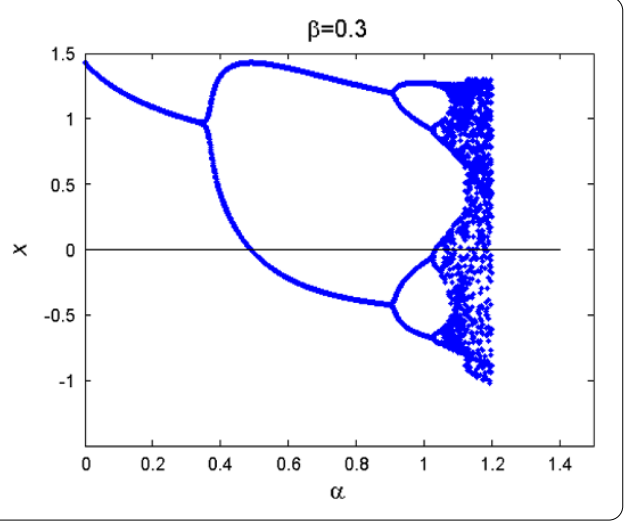

Figure 5 Bifurcation of (1.1)-(1.2) when $r_{1}=0.3$ and $r_{2}=0.1$ and $t \in[0,20]$.

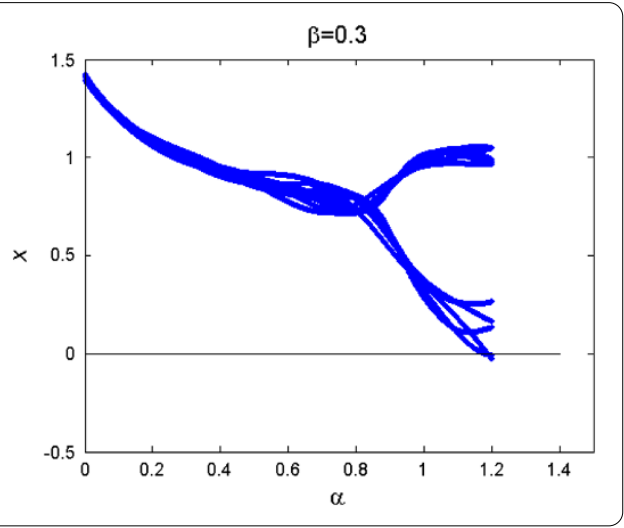

Figure 6 Bifurcation of (1.1)-(1.2) when $r_{1}=0.25$ and $r_{2}=0.15$ and $t \in[0,15]$.

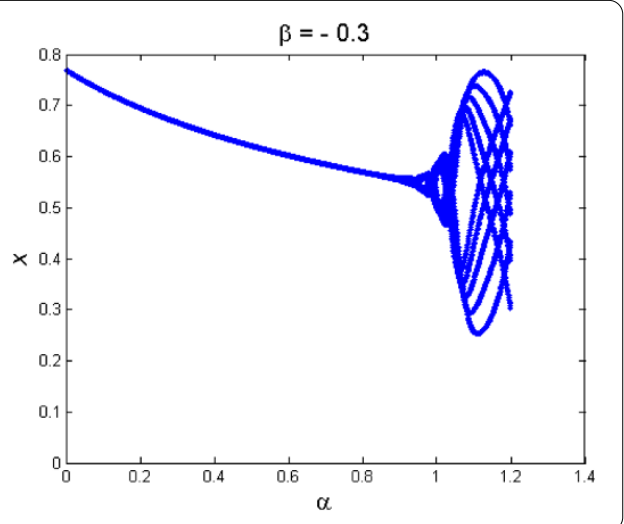


We see clearly in Figure 3 the bifurcation from a stable fixed point to a stable orbit of period two at $\alpha=0.4$, and then the bifurcation from period two to period four at $\alpha=0.9$. The further period doubling occurs at decreasing increments in $\alpha$, and the orbit becomes chaotic for $\alpha \simeq 1.1$.

- Case 2: $r_{1}=r_{2}$.

Take $r_{1}=r_{2}=1$ and $t \in[0,100]$ in (1.1)-(1.2) (Figure 7).

Take $r_{1}=r_{2}=2$ and $t \in[0,200]$ in (1.1)-(1.2) (Figure 8).

Take $r_{1}=r_{2}=0.1$ and $t \in[0,10]$ in (1.1)-(1.2) (Figure 9).

Take $r_{1}=r_{2}=0.2$ and $t \in[0,15]$ in (1.1)-(1.2) (Figure 10).

Figure 7 Bifurcation of (1.1)-(1.2) when $r_{1}=r_{2}=1$ and $t \in[0,100]$.

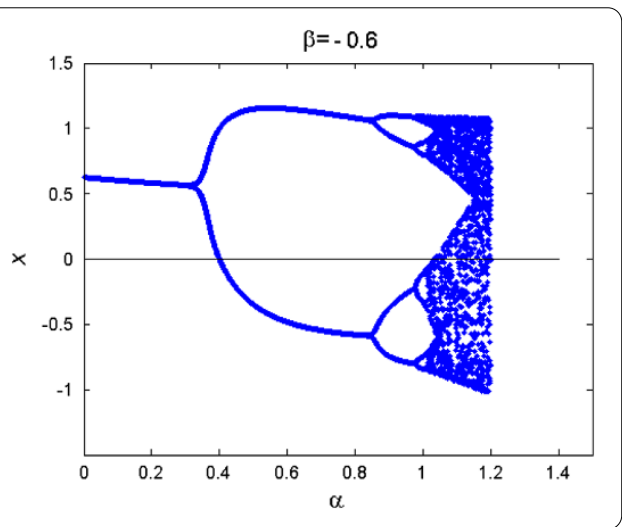

Figure 8 Bifurcation of (1.1)-(1.2) when $r_{1}=r_{2}=2$ and $t \in[0,200]$.

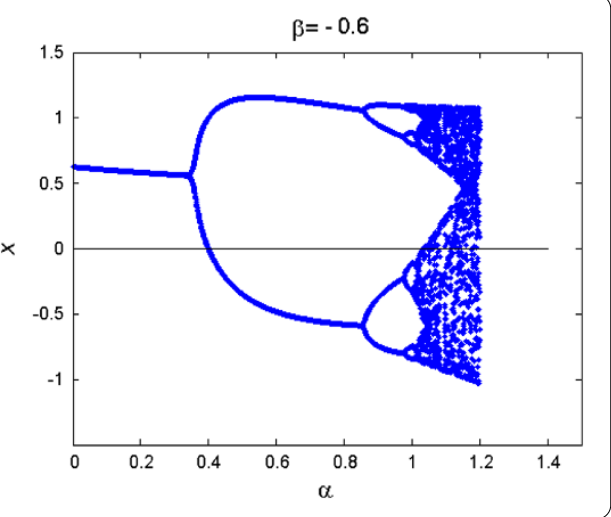

Figure 9 Bifurcation of (1.1)-(1.2) when $r_{1}=r_{2}=0.1$ and $t \in[0,10]$.

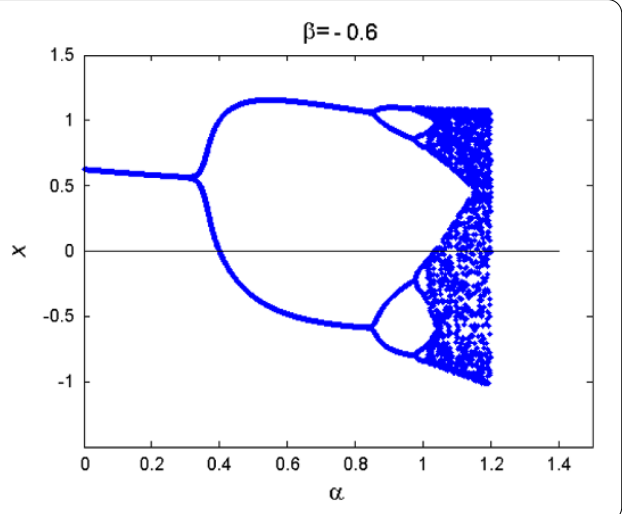




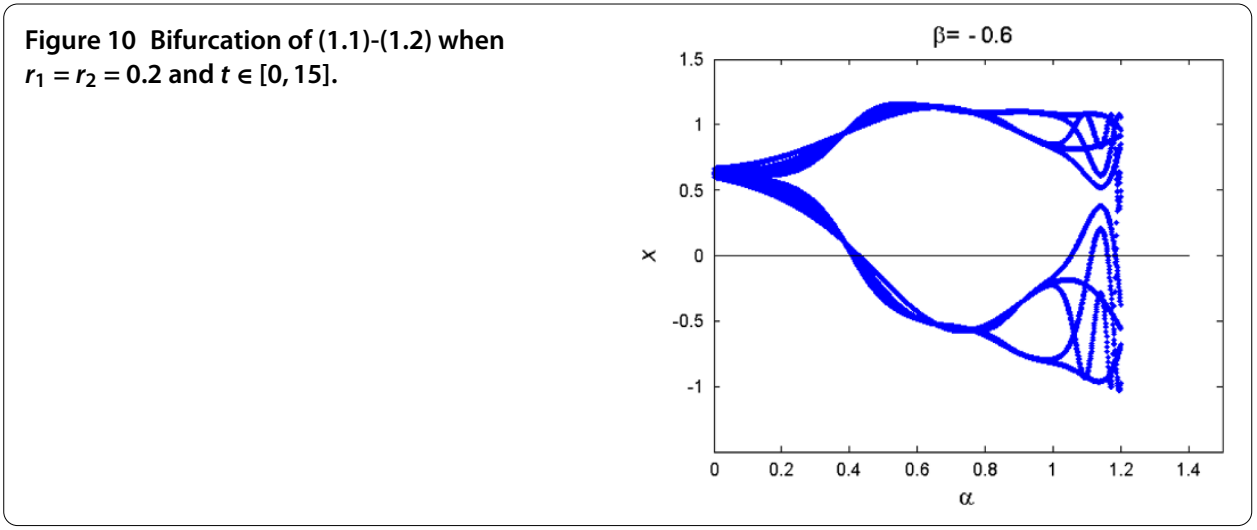

Figure 11 Bifurcation of (1.1)-(1.2) when $r_{1}=1$ and $r_{2}=2$ and $t \in[0,200]$.

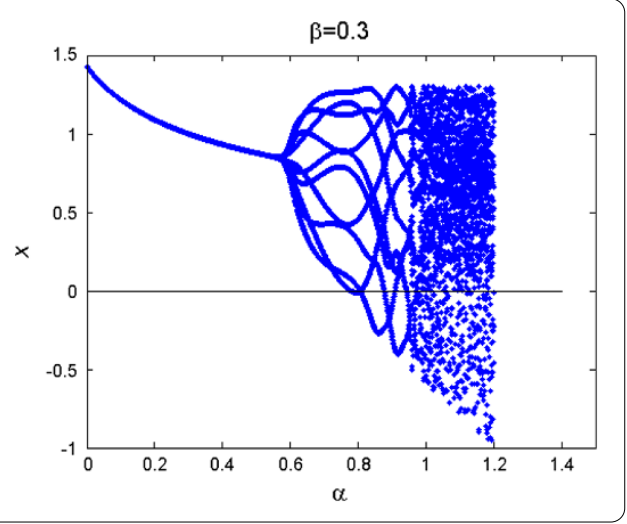

Figure 12 Bifurcation of (1.1)-(1.2) when $r_{1}=0.1$ and $r_{2}=0.2$ and $t \in[0,20]$.

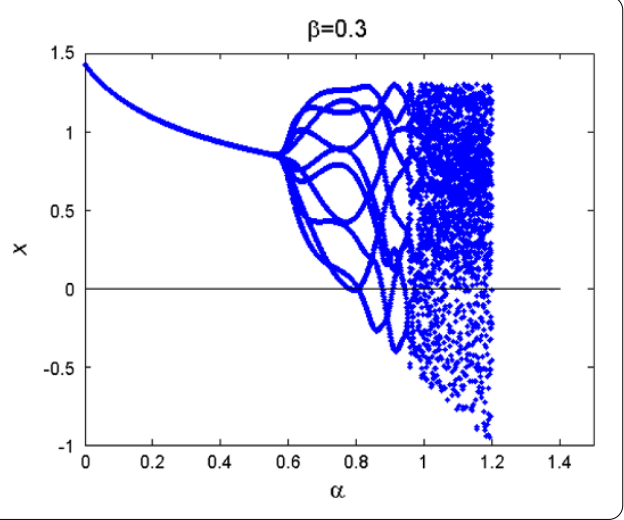

- Case 3: $r_{1}<r_{2}$.

Take $r_{1}=1$ and $r_{2}=2$ and $t \in[0,200]$ in (1.1)-(1.2) (Figure 11).

Take $r_{1}=0.1$ and $r_{2}=0.2$ and $t \in[0,20]$ in (1.1)-(1.2) (Figure 12).

Take $r_{1}=0.25$ and $r_{2}=0.75$ and $t \in[0,30]$ in (1.1)-(1.2) (Figure 13).

Take $r_{1}=0.15$ and $r_{2}=0.25$ and $t \in[0,50]$ in (1.1)-(1.2) (Figure 14).

\section{Chaotic attractor}

In this section we are interested in studying the chaotic attractor for three different cases.

- Case 1: $r_{2}>r_{1}$. 


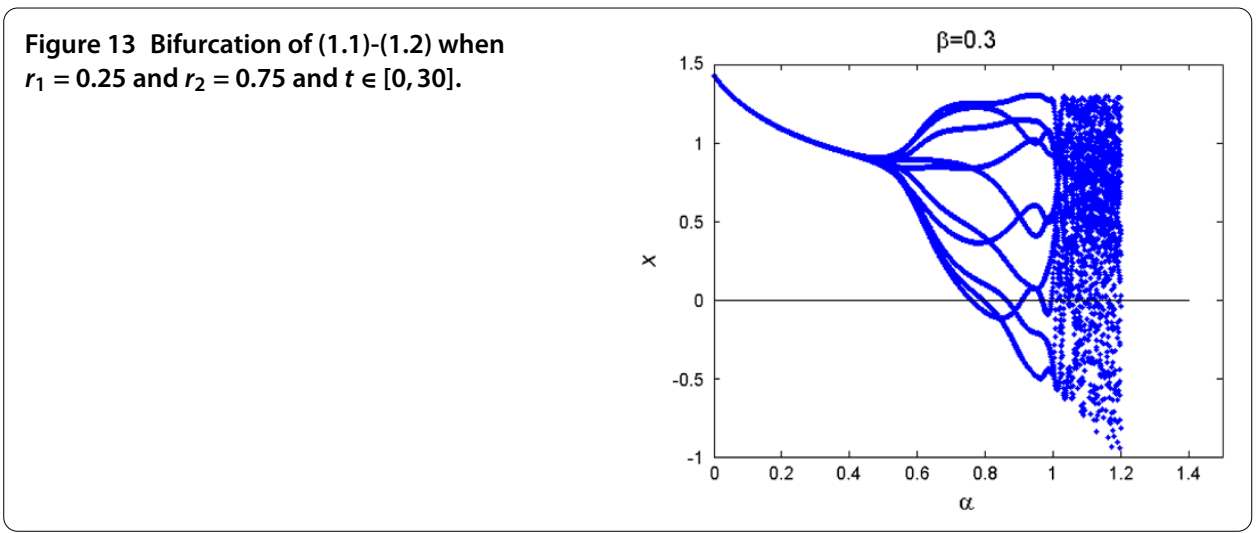

Figure 14 Bifurcation of (1.1)-(1.2) when $r_{1}=0.15$ and $r_{2}=0.25$ and $t \in[0,50]$.

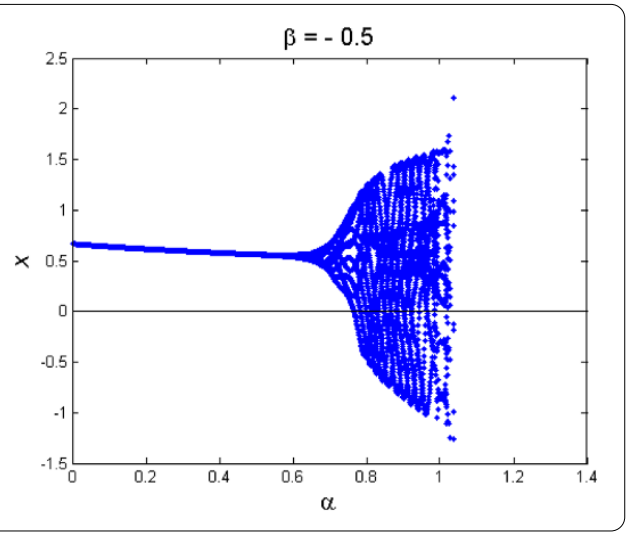

Figure 15 Chaotic attractor of (5.1)-(5.2).

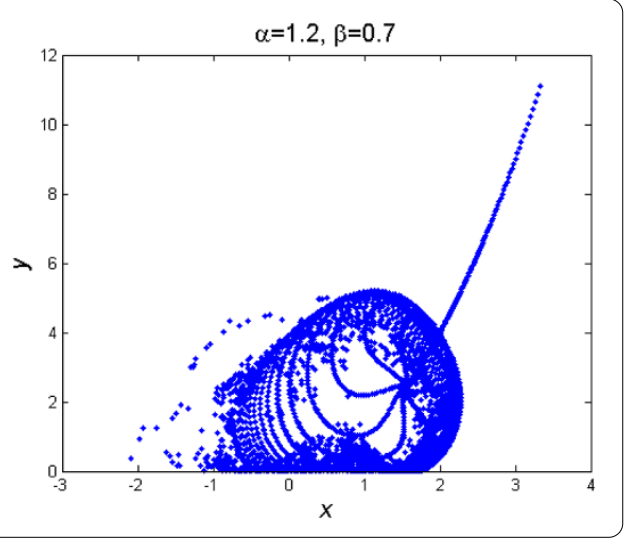

Here we rewrite system (1.1)-(1.2) as follows:

$$
\begin{aligned}
& x(t)=1+\beta x\left(t-r_{2}\right)-\alpha y\left(t-r_{2}\right), \\
& y(t)=x^{2}\left(t-\left(r_{1}-r_{2}\right)\right) .
\end{aligned}
$$

It is worth here to mention what we get when we plot the chaotic attractor for system (5.1)-(5.2) in this case. Figure 15 shows the chaotic attractor when $r_{1}=2$ and $r_{2}=1$, while Figure 16 shows the chaotic attractor of the same when $r_{1}=0.25$ and $r_{2}=0.15$.

- Case 2: $r_{1}=r_{2}=r$. 


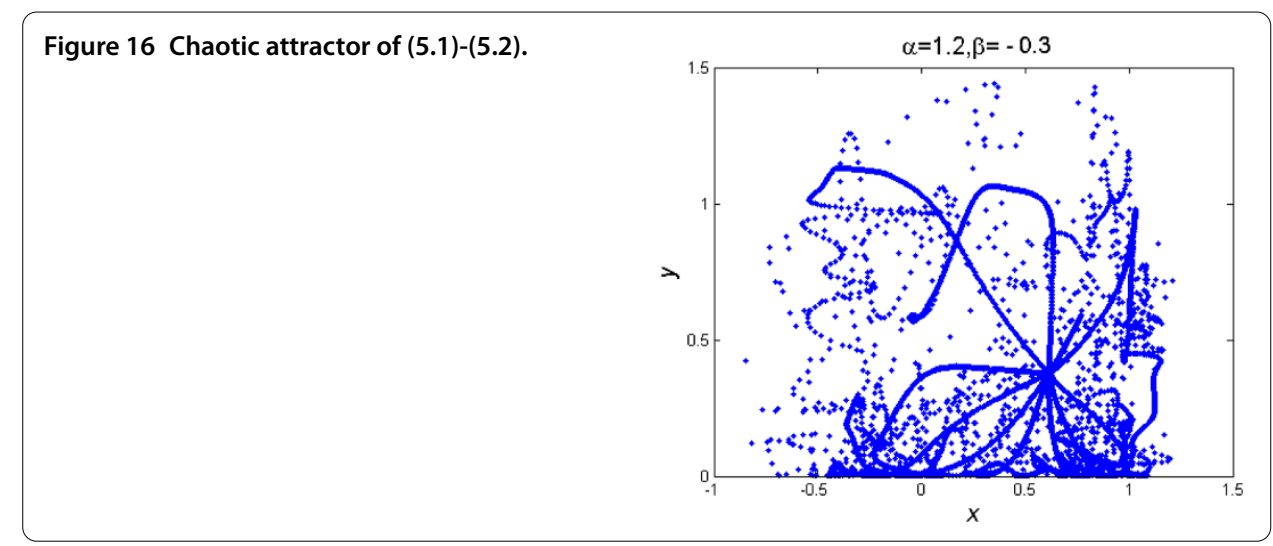

Figure 17 Chaotic attractor of (5.3)-(1.2) with

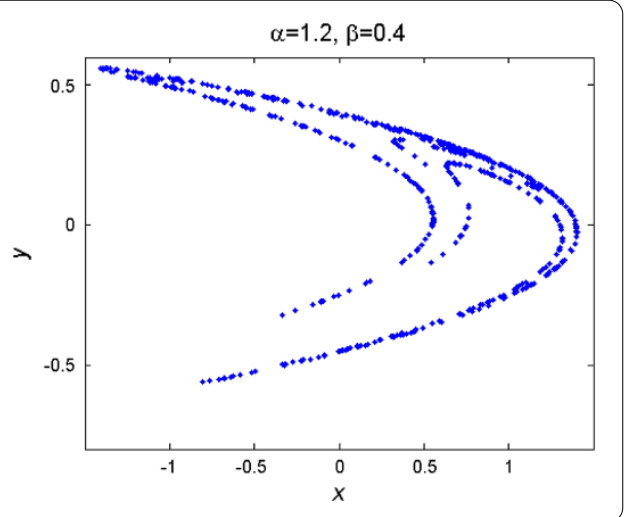
$r_{1}=r_{2}=1, t \in[0,75]$.

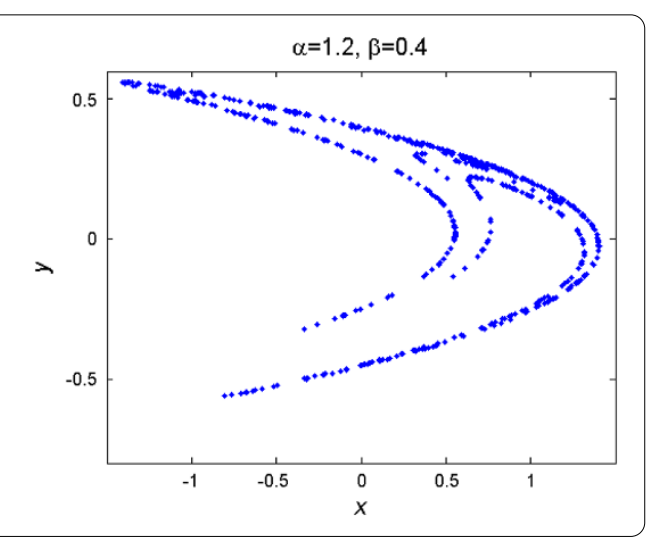

Figure 18 Chaotic attractor of (5.3)-(1.2) with $r_{1}=r_{2}=0.1, t \in[0,7.5]$.

Here system (1.1)-(1.2) is rewritten as

$$
x(t)=1-\alpha x^{2}(t-r)+\beta x(t-r)
$$

with

$$
x(t)=x_{0}, \quad t \leq 0 .
$$

In this case, the chaotic attractor for $r_{1}=r_{2}=1$ and $r_{1}=r_{2}=0.1$ looks like in Figures 17 and 18.

- Case 3: $r_{1}>r_{2}$. 


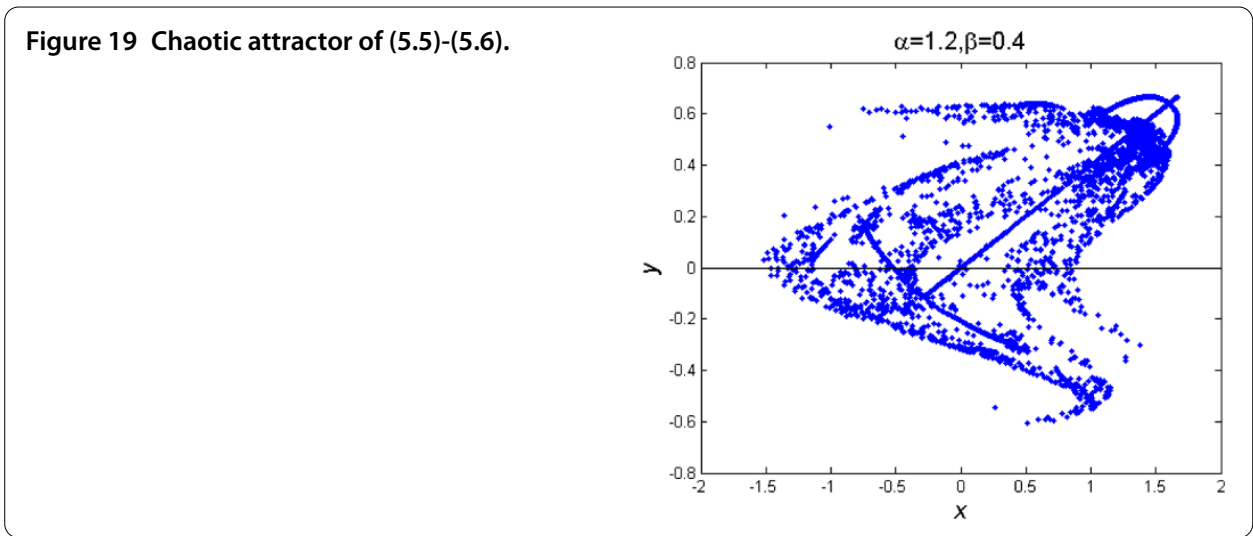

Figure 20 Chaotic attractor of (5.5)-(5.6).

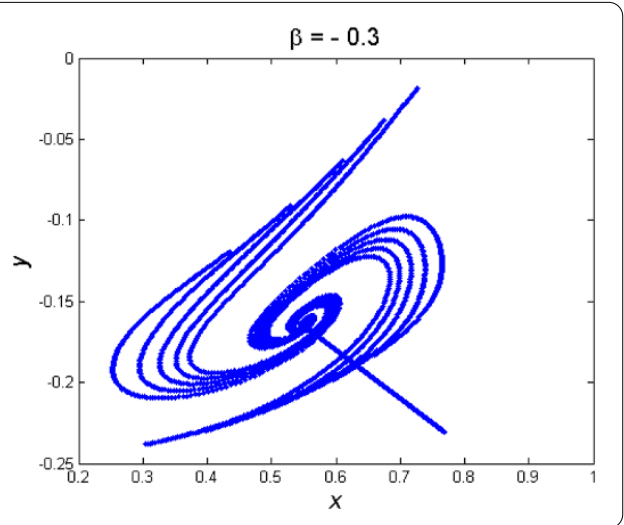

Here we also rewrite system (1.1)-(1.2) as follows:

$$
\begin{aligned}
& x(t)=1-\alpha x^{2}\left(t-r_{1}\right)+y\left(t-r_{1}\right), \\
& y(t)=\beta x\left(t-\left(r_{2}-r_{1}\right)\right) .
\end{aligned}
$$

Here we show the chaotic attractor for system (5.5)-(5.6). Figure 19 shows the chaotic attractor when $r_{1}=1$ and $r_{2}=2$, while Figure 20 shows the chaotic attractor of the same system but with $r_{1}=0.15$ and $r_{2}=0.25$.

Since the Lyapunov exponent is a good indicator for the existence of chaos [18-21], we compute the Lyapunov characteristic exponents (LCEs) via the Householder QR-based methods described in [22]. LCEs play a key role in the study of nonlinear dynamical systems, and they are a measure of sensitivity of solutions of a given dynamical system to small changes in the initial conditions. One feature of chaos is sensitive dependence on initial conditions; for a chaotic dynamical system, at least one LCE must be positive. Since for non-chaotic systems all LCEs are non-positive, the presence of a positive LCE has often been used to help determine if a system is chaotic or not. Figure 21 shows the LCEs for system (1.1)-(1.2) in the case $r_{1}>r_{2}$ for $\beta=0.3$ with the initial conditions $\left(x_{0}, y_{0}\right)=(0,0)$. With these parameter values, we find that LCE1 $=0.3228$ and LCE2 $=-1.2461$. While Figure 22 shows the LCEs for the same system in the case $r_{1}<r_{2}$ for $\beta=-0.5$ with the same initial conditions, we find that LCE1 $=0.1318$ and LCE2 $=-0.8153$. Finally, Figure 23 shows the LCEs for system (1.1)-(1.2) in the case $r_{1}=r_{2}$ for parameter values $\beta=-0.6$ with the same initial conditions. We find that LCE1 $=0.3228$ and LCE $2=-1.2460$. 
Figure 21 LCEs of (5.1)-(5.2) when $r_{1}>r_{2}$.

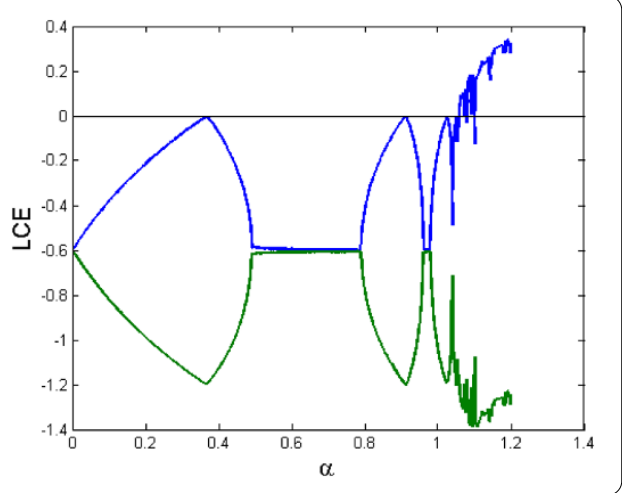

Figure 22 LCEs of (5.1)-(5.2) when $r_{1}<r_{2}$.

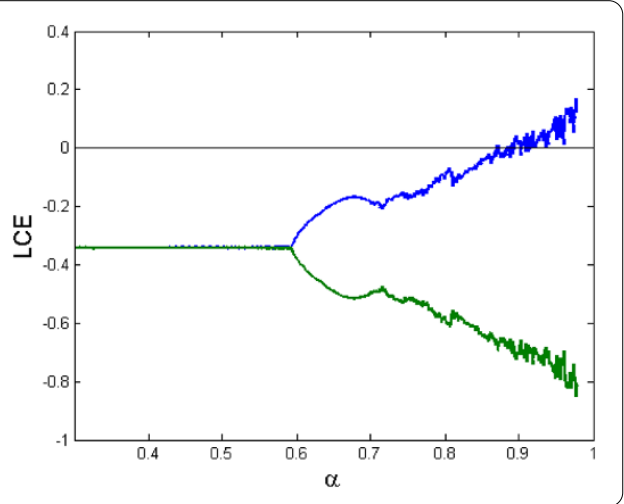

Figure 23 LCEs of (5.1)-(5.2) when $r_{1}=r_{2}$.

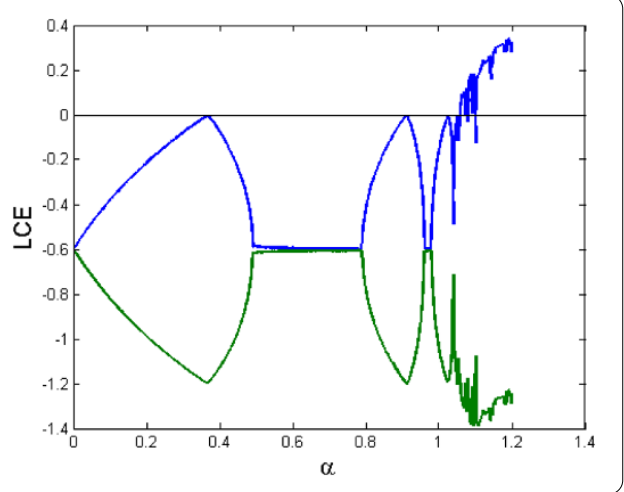

\section{Conclusion}

The discontinuous (sectionally continuous) dynamical system of the Henon map describes dynamical properties for different values of the parameters $r_{1}, r_{2} \in \mathbb{R}^{+}$when the time $t \in[0, T]$ is continuous. Indeed, the stability of fixed points depends on the values of delay parameters $r_{1}$ and $r_{2}$ as we have seen. The bifurcation diagrams, as well, depend on the values of delay parameters $r_{1}$ and $r_{2}$ and the time interval $[0, T]$. We have also noticed that the chaotic attractor of the discontinuous (sectionally continuous) Henon system in its different versions is also affected by the change in $r_{1}, r_{2}$ and the time interval $[0, T]$. On the other hand, from Figures 3-4, 7-8, and 11-12 it looks like there is a scale that gives identical chaotic behavior. To summarize, our analytical result (3.1) agrees with the numerical simulations. 


\section{Competing interests}

The authors declare that they have no competing interests.

\section{Authors' contributions}

The authors declare that the study was realized in collaboration with the same responsibility. All authors read and approved the final manuscript.

\section{Author details}

${ }^{1}$ Department of Mathematics, Faculty of Science, Alexandria University, Alexandria, Egypt. ${ }^{2}$ Faculty of Education, Alexandria University, Alexandria, Egypt.

\section{Acknowledgements}

The authors would like to thank the referees of this manuscript for their valuable comments and suggestions.

\section{Received: 9 June 2013 Accepted: 24 March 2014 Published: 08 Apr 2014}

\section{References}

1. El-Sayed, AMA, Nasr, ME: Existence of uniformly stable solutions of nonautonomous discontinuous dynamical systems. J. Egypt. Math. Soc. 19(1-2), 91-94 (2011)

2. El-Sayed, AMA, Nasr, ME: On some dynamical properties of discontinuous dynamical systems. Am. Acad. Sch. Res. J. 2(1), 28-32 (2012)

3. El-Sayed, AMA, Nasr, ME: Dynamic properties of the predator-prey discontinuous dynamical system. Z. Naturforsch. A $67 a, 57-60(2012)$

4. El-Sayed, AMA, Nasr, ME: On some dynamical properties of the discontinuous dynamical system presents the logistic equation with different delays. i-manag. J. Math. 1(1) (2012)

5. El-Sayed, AMA, Nasr, ME: Some dynamic properties of a discontinuous dynamical system. Alex. J. Math. 3(1) (2012)

6. El-Sayed, AMA, Nasr, ME: Discontinuous dynamical systems and fractional-orders difference equations. J. Fract. Calc. Appl. 4(12), 1-9 (2013)

7. El-Sayed, AMA, Salman, SM: Chaos and bifurcation of discontinuous dynamical systems with piecewise constant arguments. Malaya J. Mat. 1(1), 15-19 (2012)

8. El-Sayed, AMA, Salman, SM: Chaos and bifurcation of discontinuous logistic dynamical system with piecewise constant arguments. Malaya J. Mat. 3(1), 14-20 (2013)

9. El-Sayed, AMA, El-Raheem, ZF, Salman, SM: On some dynamics of Duffing dynamical system generated by a semi-discretization process with two different delays. Math. Sci. Lett. 3(2), 89-96 (2014)

10. El-Sayed, AMA, Salman, SM: Discontinuous dynamical systems generated by a semi-discretization process. Electron. J. Math. Anal. Appl. 1(1), 47-54 (2013)

11. Bilal, S, Ramaswamy, R: The generalized time-delayed Henon map: bifurcations and dynamics. Int. J. Bifurc. Chaos 23(3), 1350045 (2013)

12. Sprott, JC: High-dimensional dynamics in the delayed Henon map. Electron. J. Theor. Phys. 3(12), 19-35 (2006)

13. Bohai, NA: Continuous solutions of systems of nonlinear difference equations with continuous arguments and their properties. Nonlinear Oscil. 10(2), 169-175 (2007)

14. da Cruze, JH, Táboas, PZ: Periodic solutions and stability for a singularly perturbed linear delay differential equation. Nonlinear Anal. 67, 1657-1667 (2007)

15. Elaydi, SN: An Introduction to Difference Equations, 3rd edn. Undergraduate Texts in Mathematics. Springer, New York (2005)

16. Hale, J: Theory of Functional Differential Equations. Springer, New York (1977)

17. Kuznetsov, YA: Elements of Applied Bifurcation Theory, 3rd edn. Springer, Berlin (2004)

18. Jing, ZJ, Yang, J: Bifurcation and chaos in discrete-time predator-prey system. Chaos Solitons Fractals 27, 259-277 (2006)

19. Liu, X, Xiao, D: Complex dynamic behaviors of a discrete-time predator-prey system. Chaos Solitons Fractals 32, 80-94 (2007)

20. Wu, G-C, Baleanu, D: Discrete fractional logistic map and its chaos. Nonlinear Dyn. 75(1-2), 283-287 (2014)

21. Wu, G-C, Baleanu, D, Zeng, S-D: Discrete chaos in fractional sine and standard maps. Phys. Lett. A (2014). doi:10.1016/j.physleta.2013.12.010

22. Udwadia, $\mathrm{FE}$, von Bremen, $\mathrm{H}$ : A note on the computation of the largest $p$-Lyapunov characteristic exponents for nonlinear dynamical systems. J. Appl. Math. Comput. 114, 205-214 (2000) 